\title{
Relationship between Facebook Addiction, Depression and Shyness among college students in Mumbai
}

\author{
Ruchi Kulkarni ${ }^{1}$, Anuja Deshpande ${ }^{2}$ \\ ${ }^{1} 2^{\text {nd }}$ year, Master of Arts in Clinical Psychology, Maniben Nanavati Women's College, Mumbai. \\ ${ }^{2}$ Assistant Professor and Post Graduate In Charge, Department of Psychology, Maniben Nanavati \\ Women's College, Mumbai \\ Corresponding author: Anuja Deshpande \\ Email: anuja185@gmail.com
}

\begin{abstract}
Background: Our personal life is highly dependent on the technology that has been developed. It has changed the way one lives, communicates and interacts with other people. As people's demands and life style have changed, the demand for advancing of the technology has also increased. One type of technological advancement which has become an integral part of everyday life are mobile phones. The present study intends to examine the relationship between Facebook addiction, depression and shyness among college students in Mumbai.

Methods: This study was conducted in various areas of Mumbai, focusing on Facebook users among college students The participants were given a form for demographic details, a consent form, and a copy of the Bergen Facebook Addiction Scale (BFAS) Becks Depression Inventory II (BDI II) and Revised Cheek and Buss Shyness Scale (RCBSS) Codes were assigned to each participant to maintain their anonymity. The data collection was done in person.

Results: Facebook addiction showed significant positive correlation with shyness with the $\mathrm{r}$ value was 0.199 which was significant at 0.01 level, i.e. $(r=0.199, \mathrm{p}<.05)$ Also Facebook addiction showed positive correlation with depression with the $r$ value was 0.334 which was significant at 0.01 level, i.e. $(r=$ $0.334, \mathrm{p}<.01)$.

Conclusion: A significant positive correlation has been found between Facebook addiction and shyness and Facebook addiction and depression among the sample of study.
\end{abstract}

Keywords: Facebook addiction, depression, shyness, students.

(Paper received $-9^{\text {th }}$ January 2019, Peer review completed $-20^{\text {th }}$ March 2019)

(Accepted $-24^{\text {th }}$ March 2019)

\section{INTRODUCTION}

Development of mobile phones made it easy to communicate messages from one place to other. It made the communication efficient and made it faster. As the demand for mobile phones increased there came better versions which included better features. Today's most widely used phones are smartphones based on android, windows and IOS system which gives rise to social media usage.

Social media includes websites and applications that enable its users to create and share content or to participate in social networking. It has empowered communication and interaction with people all over the globe. It facilitates the sharing of information, ideas, career interests and other forms of expression via virtual communities and networks. There are various social media sites which are in demand some of them are Practically all college undergraduate students are Facebook users, with over $90 \%$ participation being reported in some studies [1]. Since the conception of this social networking tool, college undergraduates have been using Facebook with increasing frequency, now spending around 40 minutes per day actively 
using its features [2]. There are potentially positive effects associated with the widespread use of Facebook in college students. The college student typically uses Facebook as a method of keeping in touch with old and current friends, and to post, share and look at pictures, as opposed to simply follow a current trend or to occupy free time [3].

Besides this positive and meaningful use of Facebook, spending time on the site has been associated with increased life satisfaction, social trust, and political participation in college students [4]. It is apparent that Facebook has become integrated into the daily lives of undergraduates, although some may not consider this form of social interaction to be beneficial to the learning environment colleges strive to promote.

Facebook being one of the most used and popular social media sites there have been concerns regarding its addictive use which hampers the psychophysiological health of the person. Facebook addiction is one of the most growing concerns in today's lifetime. It is a form of social media addiction in which the excessive use of Facebook hampers the daily life functioning of an individual. The researches indicate addiction was positively connected to psychological factors like depression, and negatively connected to self-esteem and life satisfaction [5]. Because of the lack of evidence, the broader perspective of internet addiction is taking into account to gain more insights and evidence for behavioral addiction. Because of the similar symptoms of internet and Facebook addiction the two online platforms have an overlap especially on a social level because of the communication possibilities within both platforms.

\section{Depression}

Depression comes from the Latin word "depressare" and the classical Latin word "deprimere". Deprimere literally means "press down". de translates into "down" and premere translates into "to press." In essence, the term appears to denote a feeling of heaviness, of being "pressed down," that is also referred to as "sad," "blue," or simply "down." Depression (major depressive disorder) is a common and serious medical illness that negatively affects how you feel, the way you think and how you act. Fortunately, it is also treatable. Depression causes feelings of sadness and/or a loss of interest in activities once enjoyed. It can lead to a variety of emotional and physical problems and can decrease a person's ability to function at work and at home. The depressive symptoms can range from mild to severe where it includes feeling sad or being in depressed mood. Depressed mood is a state of low mood and aversion to activity that affects the persons thoughts, behaviour and his sense of well-being. There is also loss of interest in activity which was earlier enjoyed by the person. One of the peculiar symptoms that are seen in depression are changes in appetite, where the person either eats a lot or doesn't feel like eating at all. This leads to weight gain or weight loss.

Other concern is in regard to sleep. Where the person may experience insomnia where there is lack of sleep or hypersomnia where the person sleeps a lot. Along with changes in the sleep patterns and changes in the appetite there is also loss of energy or increased energy seen. Here the person is either doesn't have the energy to do the activity or feels a lot of energy and feels like constantly doing some or the other activity.

Along with the physiological symptoms there are also psychological symptoms which are experienced by the person. One of the symptoms is feeling worthless or guilty. Individuals who feel worthless may feel insignificant, useless, or believe they have nothing valuable to offer the world. They also experience guilt where you feel bad about what you have done or who you are. These feeling combined with the physiological symptoms also lead the person to have thoughts of death or suicide. When there are thoughts of suicide the person is constantly preoccupied with the thoughts of suicide. The range of suicidal ideation can vary from having just fleeting thoughts to actually having extensive thoughts. These symptoms must last at least two weeks for a diagnosis of depression.

\section{Shyness}

Shyness has been defined as "a heightened state of individuation characterized by excessive egocentric preoccupation and overconcern with social evaluation, with the consequence that the shy person inhibits, withdraws, avoids, and escapes" social interactions [6]. themselves to social situations, people who experience shyness often avoid them altogether. There are some shyness eliciting situations which may increase an individual's level of shyness. Research suggests that the most powerful shyness eliciting 
situations involve interactions with authority figures, one to one encounters with members of the opposite sex for heterosexuals, conversations with strangers, being the focus of attention in small groups and explicitly evaluative situations, such as job interviews and public speaking [7-8]. Shy people tend to regard their networks as less supportive and less satisfying and are happy and more comfortable to be by themselves [9]. Research has also showed that the computer-mediated medium provides shy people a perfect environment to perceive control over the communication process [10].

Izard (1972) described shyness as a discrete, fundamental emotion. An emotion profile in a "shy" situation includes interest and fear, which interacts with shyness [11]. Carver and Scheier (1986) defined shyness in self-regulation terms, with unfavourable social outcome expectancies leading to disengagement in task efforts. There are various symptoms of shyness which include cognitive, physical, emotional and psychological symptoms. Cognitive Symptoms of Shyness: Thoughts play a crucial role in shyness. When confronted with a trigger, many automatic thoughts may be activated. A shy person's thoughts are likely to be self-focused, involve some kind of negative anticipatory outcome and be focused on what others may be thinking [12]. There are self-deprecating thoughts which are usually accompanied by physical discomfort. Automatic thoughts may cause physical symptoms to occur. Individuals may physical symptoms such as racing heart, shortness of breath, dry mouth, sweating, muscle tension and trembling.

The combination of negative self-talk and physical symptoms typically leads to distressing and hurtful feelings. A shy person is likely to experience feelings such as shame and embarrassment. A shy person may also experience guilt and self-contempt. For some people these negative experiences can lead to anger and frustration towards themselves selves or others. Individuals who are shy also often experience selfconsciousness. A shy person may become more acutely aware of their own body sensations and their own feelings of inadequacy.

Along with physical and emotional symptoms there are also behavioural symptoms experienced. The most common behaviour is avoidance. Because of the pain of exposing themselves to social situations, people who experience shyness often avoid them altogether. There are some shyness eliciting situations which may increase an individual's level of shyness. Research suggests that the most powerful shyness eliciting situations involve interactions with authority figures, one to one encounters with members of the opposite sex for heterosexuals, conversations with strangers, being the focus of attention in small groups and explicitly evaluative situations, such as job interviews and public speaking [6-8]. Shy people tend to regard their networks as less supportive and less satisfying and are happy and more comfortable to be by themselves [9]. Research has also showed that the computer-mediated medium provides shy people a perfect environment to perceive control over the communication process [10].

\section{METHODOLOGY}

\section{Research Questions}

The following are the research questions for the study:

1. Has Facebook addiction contributed to depression among college students in Mumbai?

2. Has Facebook addiction contributed to shyness among college students in Mumbai?

Aim : To determine the relation of Facebook addiction, depression and shyness among college students in Mumbai.

\section{Objectives}

1. To find out the association between Facebook addiction and Depression among college students

2. To find out the association between Facebook addiction and shyness among college students

\section{Hypotheses}

Following were the hypotheses for the study:

1. There will be a significant relation between Facebook addiction and shyness.

2. There will be significant relation between Facebook addiction and depression. 


\section{Variables}

The variables under investigation were:
1. Facebook addiction
2. Depression
3. Shyness

\section{Operational definitions}

- Facebook Addiction: Facebook addiction was measured by scores obtained on the Bergen Facebook Addiction Scale given by Dr Cecilie Andraessen, Torjorn Torsheim, Geir Brunborg and Stale Pallesen(2012)

- Depression: Depression was measured by the scores obtained on the scale of Becks Depression Inventory II given by Aron Beck (1978)

- Shyness: Shyness was measured by scores obtained on Revised Cheek and Buss Shyness scale by Jonathon Cheek (1983)

Sample size: The sample consisted of 120 college students who were from Mumbai between the age range of 18 to 25 years having an active Facebook account. with mean of 19.62 and S.D 1.583

\section{Inclusion Criteria}

1. College students with active Facebook account

2. College students above age of 18 years

3. College students who are familiar with English language

4. Students from colleges in Mumbai

\section{Exclusion Criteria}
1. College students without an active Facebook account
2. College students with no Facebook account
3. Students from other colleges outside Mumbai
4. College students who are unfamiliar with English language.
5. College students below age of 18 years
6. College students above age of 25 years

\section{Research Design}

A quantitative correlation study was employed using survey method. A correlational study was used to find if there exists a relationship between two variables. It studied whether an increase or decrease in one variable corresponds to an increase or decrease in the other variable.

\section{Sampling Method}

A non-probability sampling technique was used in this study. Convenience sampling was used which is a type of non-probability sampling technique in which subjects were selected because of their convenient accessibility and proximity to the researcher.

\section{Scales Used}

1. Bergen Facebook Addiction Scale (BFAS): It is a 18 item likert scale, ranging from 1(very rarely) to 5 (very often). The scoring is done by summing up the responses. Higher the scores more the addiction. The 18 items reflect each of the six core elements of addiction (salience, mood modification, tolerance, withdrawal, conflict, and relapse), and were administered to 423 students together with several other standardized self-report scales (Addictive Tendencies Scale, Online Sociability Scale, Facebook Attitude Scale, NEO-FFI, BIS/BAS scales, and Sleep questions). The factor structure of the scale was good $($ RMSEA $=0.046$, CFI $=0.99)$ and coefficient alpha was 
0.83 . The 3-week test-retest reliability coefficient was 0.82 . The scores converged with scores for other scales of Facebook activity. Also, they were positively related to Neuroticism and Extraversion, and negatively related to Conscientiousness.

2. Becks Depression Inventory II: It is a self-report measure of depression consisting of 21 items. It is a Likert type scale ranging from 0 to 3 . The total score ranges from 0 to 63 . High concurrent validity ratings were given between the BDI and other depression instruments as the Minnesota Multiphasic Personality Inventory and the Hamilton Depression Scale, 0.77 correlation rating was calculated when compared with inventory and psychiatric ratings. The BDI-II positively correlated with the Hamilton Depression Rating Scale, $r=0.71$, had a one-week test-retest reliability of $r=0.93$ and an internal consistency $\alpha=0.91$.

3. Revised Cheek and Buss Shyness Scale: It is a 13-item scale measuring shyness. The responses are presented in Likert type format and the responses range from 1 (strongly disagree) to 5(strongly agree). Reliability was computed using Cronbach's $\alpha$ was 0.83 , validity coefficients were calculated using the Pearson product-moment correlation coefficient (r) statistic. The correlation of the items with the total scale was adequate, with the lowest correlation occurring in item $3(r=0.35)$.

\section{STATISTICAL ANALYSIS}

The data was analysed using Descriptive statistics for the variables and for age of the participants. To further verify the hypotheses Pearson Product Moment Correlation was computed. Regression analysis was also carried out to for predict Facebook addiction through shyness and depression

\section{RESULTS}

The main aim of the study was conducted to find the relation between Facebook addiction, depression and shyness among college students in Mumbai. The sample size was 120 with both males and females.

After data collection, the next step is to analyse the data statistically and interpret the findings at both statistical and theoretical levels. Collected data has been presented in a table form after calculating basic statistical factors like mean, median, standard deviation.

The mean for Facebook Addiction Scale was 33.52 with SD 13.83. The mean for Revised Buss and Shyness Scale was 35.94 with SD 8.60. The mean for Becks Depression Inventory II was 12.58 with SD 10.06. A scatter plot was done to see if there is linear relationship between the variables. Initially a correlation analysis was done to find out if Facebook addiction was significantly related to depression and shyness. (as reported in table 1). The Pearson correlation was done to find out the correlation. The Pearson correlation coefficient, $r$, can take a range of values from +1 to -1 . A value of 0 indicates that there is no association between the two variables. A value greater than 0 indicates a positive association; that is, as the value of one variable increases, so does the value of the other variable. A value less than 0 indicates a negative association; that is, as the value of one variable increases, the value of the other variable decreases.

The correlational and predictive trends shared by Facebook addiction with shyness, and depression were explored. Facebook addiction showed significant positive correlation with shyness. As given in the table 1 the $\mathrm{r}$ value was 0.199 which was significant at 0.01 level, i.e. $(r=0.199, \mathrm{p}<.05)$ Also Facebook addiction showed positive correlation with depression. As given in the table 1 the $\mathrm{r}$ value was 0.334 which was significant at 0.01 level, i.e. $(\mathrm{r}=0.334, \mathrm{p}<.01)$. The results revealed that Facebook addiction had an association with shyness and depression. Though the association among the variables are fairly moderate but are significant. Thus, the hypotheses that there is significant relation between Facebook addiction and shyness and there is significant relation between Facebook addiction and depression were validated. 
Table 1: Correlation Analysis between Facebook addiction, depression and shyness

\begin{tabular}{|c|l|c|c|c|}
\hline & & FAS & RBSS & BDI2 \\
\hline \multirow{3}{*}{ FAS } & Pearson Correlation & 1 & $.199^{*}$ & $.334^{* *}$ \\
\hline & Sig. (2-tailed) & & .029 & .000 \\
\cline { 2 - 5 } RBSS & $\mathrm{N}$ & 120 & 120 & 120 \\
\hline \multirow{3}{*}{ BDI2 } & Pearson Correlation & $.199^{*}$ & 1 & $.459^{* *}$ \\
\cline { 2 - 5 } & Sig. (2-tailed) & .029 & & .000 \\
\hline & $\mathrm{N}$ & 120 & 120 & 120 \\
\hline & Pearson Correlation & $.334^{* *}$ & $.459^{* *}$ & 1 \\
\cline { 2 - 5 } & Sig. (2-tailed) & .00 & .000 & \\
\hline & $\mathrm{N}$ & 0 & & 120 \\
\hline
\end{tabular}

Then a series of Regression Analysis were then conducted to estimate the extent to which Facebook addiction could be predicted from depression and shyness. The results show that shyness can predict Facebook addiction significantly $\left(\mathrm{R}=0.199, \mathrm{R}^{2}=0.40, \mathrm{~F}(4.86=0.00\right.$, sig). On the other hand, depression was able to predict Facebook addiction with significant accuracy $\left(R=0.334, R^{2}=0.112\right.$, $\mathrm{F}(14.811)=0.000$, ,sig). Thus, it seems that shyness and depression can be used to predict Facebook addiction. Details of these regression analyses are presented in Table 2.

Table 2: Regression Analysis for Facebook Addiction, Depression And Shyness

\begin{tabular}{|c|c|c|c|c|}
\hline & R & R2 & F & Sig \\
\hline Shyness & .199 & .040 & 4.860 & .000 \\
\hline Depression & .334 & .112 & 14.811 & .000 \\
\hline
\end{tabular}

\section{DISCUSSION}

The purpose of this study was to find out the relation between Facebook addiction, depression and shyness among college students of Mumbai. Social media addiction is affecting today's youth in many ways. One of the impact addictions has is on the psychological well-being of the user. Facebook is one of the most used social networking sites in India. Thus, it is important to study the psychological variables in relation with Facebook addiction.

The first hypothesis showed that there was a significant positive relation between Facebook addiction and shyness. Thus, it shows that people who score high on shyness score high on Facebook addiction. This is in line with the previous research done by Omar and Subramanian (2013) which studied direct relationships between shyness, loneliness, powerful others control, internal control and external control, Facebook addiction, the effect of Facebook usage as a mediator to the relationships and the types of gratification sought [3]. Data from 400 undergraduate students of Malaysian university using survey method. Results indicate direct relationship between all the factors except for internal control. Findings indicate three dimensions of gratifications sought are content, self-presentation and social and two of the personality characteristics shyness and loneliness are significant predictors to Facebook addiction among youths.

Similarly another research done investigated the personality trait of shyness and its relation with certain features of Facebook. 130 students were given questionnaires and the data was analysed. Results showed that shyness was significantly positively correlated with the time spent on Facebook and having favourable attitudes towards the social networking site. Shyness was significantly negatively correlated with the number of Facebook "Friends." Research has also showed that the computer-mediated medium provides shy people a perfect environment to perceive control over the communication process [10]. Thus, it is likely that they get more addicted to Facebook which is a computer mediated medium. 
In relation to the second hypothesis the results show that there is significant positive correlation between Facebook addiction and depression. Thus, it shows that people who score high on depression scale also score high on Facebook addiction scale. This is in line with the previous research done which studied the relationship between Facebook addiction, depression, anxiety, loneliness and academic performance among 150 higher secondary school \& college students. Bergen Facebook Addiction Scale, Patient Health Questionnaire, Severity Measure for Generalized Anxiety Disorder, UCLA loneliness scale were used. Correlation technique revealed that there is linear interaction between Facebook addiction and anxiety, depression. There was no correlation found between Facebook addiction, loneliness \& academic performance.

Beck (1967) believed that depression prone individuals develop a negative self-schema. They possess a set of beliefs and expectations about themselves that are essentially negative and pessimistic Thus, depressed people are more likely to get attracted to social media platform which provides them a source to portray an identity they desire to have and helps them earn social praise which helps them feel good about themselves for the time being, thus leading to excessive use of Facebook [11].

Also, according to the theory of user gratification among the needs given by the theorists, in people with depression the need for escapism from tension and unpleasant experience is likely to be present, as they desire escaping from unpleasant to pleasant, and releasing tension. This goal is attained through Facebook leading to excessive use. Even in case of people who are shy the need for social integration is satisfied through Facebook which allows them to talk to people without any apprehensions. Thus, this goal attained leads excessive use of Facebook addiction [12].

Thus, shyness and depression are positively related to Facebook addiction, also shyness and depression were found to be predictors of Facebook addiction. Thus, people who are shy or who suffer from depression are likely to be addicted to Facebook addiction.

\section{CONCLUSION}

The results of this research have been found to be in line with the hypotheses as well as past researches conducted on the relationship between Facebook addiction, depression and shyness. Thus, significant positive correlation has been found between. Facebook addiction and shyness and Facebook addiction and depression.

\section{REFERENCES}

1. Ellison NB, Steinfield C, Lampe C. The benefits of Facebook "friends:" Social capital and college students' use of online social network sites. J Comp Med Commun 2007;12(4):1143-68.

2. Muise A, Christofides E, Desmarais S. More information than you ever wanted: Does Facebook bring out the green-eyed monster of jealousy?. CyberPsychol Behav 2009;12(4):441-4.

3. Omar B, Subramanian K. Addicted to Facebook: Examining the roles of personality characteristics, gratifications sought and Facebook exposure among youths. GSTF Journal on Media and Communications (JMC) 2018;1(1).

4. Valenzuela S, Park N, Kee KF. Is there social capital in a social network site?: Facebook use and college students' life satisfaction, trust, and participation. J Comp Med Commun 2009;14(4):875-901.

5. Błachnio A, Przepiórka A, Pantic I. Internet use, Facebook intrusion, and depression: results of a crosssectional study. Eur Psychiatry 2015;30(6):681-4.

6. Zimbardo PG. Shyness and the stresses of the human connection. Handbook of stress: Theoretical and clinical aspects. 1982:466-81.

7. Cheek JM, Stahl SS. Shyness and verbal creativity. J Res Personality 1986;20(1):51-61.

8. Henderson L, Zimbardo PG. An overview of Shyness, what is known and how it is treated. Ralf Schwarzer. et al. Encyclopedia of Mental Health. 1998.

9. Parrott L. Helping your struggling teenager: A parenting handbook on thirty-six common problems. Zondervan; 2000. 
10. Leung L. Net-generation attributes and seductive properties of the internet as predictors of online activities and internet addiction. CyberPsychol Behav 2004;7(3):333-48.

11. Izard CE, Buechler S. Emotion expressions and personality integration in infancy. InEmotions in personality and psychopathology 1979 (pp. 445-472). Springer, Boston, MA.

12. Zeman A. What in the world is consciousness?. Progr Brain Res 2005;150:1-10.

Acknowledgements - Nil

Conflict of Interest - Nil

Funding - Nil 